A. GASULL and R. PROHENS (Barcelona)

\title{
EFFECTIVE COMPUTATION OF THE FIRST LYAPUNOV QUANTITIES FOR A PLANAR DIFFERENTIAL EQUATION
}

Abstract. We take advantage of the complex structure to compute in a short way and without using any computer algebra system the Lyapunov quantities $V_{3}$ and $V_{5}$ for a general smooth planar system.

1. Introduction. Consider the differential equation $(\dot{x}, \dot{y})=(f(x, y)$, $g(x, y)),(x, y) \in \mathbb{R}^{2}$, in the plane where $f$ and $g$ are analytic functions satisfying $f(0,0)=g(0,0)=0$. It is well known that when the origin is a non-hyperbolic critical point of focus type the study of its stability can be reduced to the computation of the so called Lyapunov quantities, $V_{2 k+1}, k=1,2, \ldots$; see [ALGM] for more details. By making a linear change of coordinates and a rescaling of the time variable if necessary, the planar differential equation can be written as

$$
\dot{z}=F(z, \bar{z})=i z+\sum_{k=2}^{\infty} F_{k}(z, \bar{z}),
$$

where $z=x+i y=\operatorname{Re}(z)+i \operatorname{Im}(z)$, and $F_{k}$ is a complex homogeneous polynomial of degree $k$.

In this paper we make some modifications in the standard techniques explained in $[\mathrm{ALGM}]$ to obtain the Lyapunov quantities. These modifications simplify their effective computation. The main idea is to keep the complex structure of (1) during all the process.

In Section 2 we give some preliminary results and in Section 3 we prove:

1991 Mathematics Subject Classification: 34C25, 58F14.

Key words and phrases: stability, weak focus, Lyapunov quantities.

Research of the first author partially supported by a DGICYT grant, number PB930860 . 
Theorem A. Consider the differential equation (1). Set

$$
\begin{aligned}
& F_{2}(z, \bar{z})=A z^{2}+B z \bar{z}+C \bar{z}^{2} \\
& F_{3}(z, \bar{z})=D z^{3}+E z^{2} \bar{z}+F z \bar{z}^{2}+G \bar{z}^{3} \\
& F_{4}(z, \bar{z})=H z^{4}+I z^{3} \bar{z}+J z^{2} \bar{z}^{2}+K z \bar{z}^{3}+L \bar{z}^{4} \\
& F_{5}(z, \bar{z})=M z^{5}+N z^{4} \bar{z}+O z^{3} \bar{z}^{2}+P z^{2} \bar{z}^{3}+Q z \bar{z}^{4}+R \bar{z}^{5} .
\end{aligned}
$$

Then the first Lyapunov quantities of (1) are:

(i) $\quad V_{3}=2 \pi[\operatorname{Re}(E)-\operatorname{Im}(A B)]$,

(ii) $\quad V_{5}=\frac{\pi}{3}\left[6 \operatorname{Re}(O)+\operatorname{Im}\left(3 E^{2}-6 D F+6 A \bar{I}\right.\right.$

$$
\begin{aligned}
& -12 B I-6 B \bar{J}-8 C H-2 C \bar{K}) \\
& +\operatorname{Re}\left(-8 C \bar{C} E+4 A C \bar{F}+6 A \bar{B} F+6 B \bar{C} F-12 B^{2} D-4 A C D\right. \\
& -6 A \bar{B} \bar{D}+10 B \bar{C} \bar{D}+4 A \bar{C} G+2 B C \bar{G}) \\
& \left.+\operatorname{Im}\left(6 A \bar{B}^{2} C+3 A^{2} B^{2}-4 A^{2} \bar{B} C+4 \bar{B}^{3} C\right)\right] .
\end{aligned}
$$

The above result already appears in [CGMM, FLLL, G, GW, HW], but the proof that we present is shorter and does not use any computer algebra system.

2. Preliminary results. We briefly recall the definition of the Lyapunov constants. into

In the $(r, \theta)$-polar coordinates $z \bar{z}=r^{2}, \theta=\arctan \frac{\operatorname{Im}(z)}{\operatorname{Re}(z)},(1)$ is converted or equivalently, for $r$ small enough,

$$
\frac{d r}{d \theta}=\left.\frac{\operatorname{Re}[\bar{z} F(z, \bar{z})] / r}{\operatorname{Im}[\bar{z} F(z, \bar{z})] / r^{2}}\right|_{z=r e^{i \theta}},
$$

$$
\frac{d r}{d \theta}=\frac{\sum_{k=2}^{\infty} r^{k} \operatorname{Re}\left(S_{k}(\theta)\right)}{1+\sum_{k=2}^{\infty} r^{k-1} \operatorname{Im}\left(S_{k}(\theta)\right)}=\sum_{k=2}^{\infty} R_{k}(\theta) r^{k},
$$

where $S_{k}(\theta)=\left.\bar{z} F_{k}(z, \bar{z})\right|_{z=e^{i \theta}}=e^{-i \theta} F_{k}\left(e^{i \theta}, e^{-i \theta}\right), R_{2}(\theta)=\operatorname{Re}\left(S_{2}(\theta)\right)$ and

$$
R_{k}(\theta)=\operatorname{Re}\left(S_{k}(\theta)\right)-\sum_{j=1}^{k-2} R_{k-j}(\theta) \operatorname{Im}\left(S_{j+1}(\theta)\right) \quad \text { for } k \geq 3 .
$$

Denote by $r(\theta, s)$ the solution of (2) which takes the value $s$ at $\theta=0$. Consider

$$
r(\theta, s)-s=\sum_{k=2}^{\infty} u_{k}(\theta) s^{k}, \quad \text { where } u_{k}(0)=0 \text { for } k \geq 2 .
$$

Then the stability of the origin of (1) is given by the sign of the first non-zero value $u_{k}(2 \pi)$. It is well known that the corresponding $k$ is odd (see [ALGM, p. 243]). 
Assume that $u_{k}(2 \pi)=0$ for $k=1, \ldots, 2 m$ and $u_{2 m+1}(2 \pi) \neq 0$. Then the $m$ th Lyapunov quantity is defined by $V_{2 m+1}=u_{2 m+1}(2 \pi)$.

The next result is inspired by $[\mathrm{AL}]$ and it allows us to compute the first values $u_{k}(2 \pi)$. In the sequel, we use the notation $\widetilde{f}=\widetilde{f}(\theta)=(f)^{\sim}(\theta)=$ $\int_{0}^{\theta} f(s) d s$.

Proposition 1. Given (2), the functions $u_{i}(\theta), i=2,3,4,5$, involved in its solution (4) are

$$
\begin{aligned}
u_{2}(\theta)= & \widetilde{R}_{2}, \\
u_{3}(\theta)= & \left(\widetilde{R}_{2}\right)^{2}+\widetilde{R}_{3}, \\
u_{4}(\theta)= & \left(\widetilde{R}_{2}\right)^{3}+2 \widetilde{R}_{2} \widetilde{R}_{3}+\widetilde{R_{2} R_{3}}+\widetilde{R}_{4}, \\
u_{5}(\theta)= & \left(\widetilde{R}_{2}\right)^{4}+3\left(\widetilde{R}_{2}\right)^{2} \widetilde{R}_{3}+\left(\widetilde{\left.R_{2}\right)^{2}} R_{3}+2 \widetilde{R}_{2} \widetilde{\widetilde{R}_{2} R_{3}}\right. \\
& +\frac{3}{2}\left(\widetilde{R}_{3}\right)^{2}+2 \widetilde{R}_{2} \widetilde{R}_{4}+2 \widetilde{R_{4} \widetilde{R}_{2}}+\widetilde{R}_{5} .
\end{aligned}
$$

P r o of. Direct substitution gives

$$
\sum_{k=2}^{\infty} R_{k}(\theta)[r(\theta, s)]^{k}=\sum_{k=2}^{\infty} u_{k}^{\prime}(\theta) s^{k} .
$$

By using the expression for a power series raised to some power (see [GR], for instance), whenever $k \geq 2$, we have

$$
u_{k}^{\prime}(\theta)=\sum_{m=2}^{k} R_{m}(\theta)\left[\sum_{M}\left(\left(\begin{array}{c}
m \\
a_{1} \ldots a_{k-1}
\end{array}\right)\right) u_{2}^{a_{2}}(\theta) u_{3}^{a_{3}}(\theta) \ldots u_{k-1}^{a_{k-1}}(\theta)\right],
$$

where $M=\left\{\left(a_{1}, \ldots, a_{k-1}\right) \in \mathbb{N}^{k-1}: a_{1}+\ldots+a_{k-1}=m, a_{1}+\ldots+(k-\right.$ 1) $\left.a_{k-1}=k\right\}$. Then the proof follows from judicious integration. As an example we prove the expression for $u_{4}(\theta)$. By using the previous formula we have

$$
u_{4}(\theta)=\int_{0}^{\theta}\left(R_{2}(\Psi)\left(2 u_{3}(\Psi)+u_{2}^{2}(\Psi)\right)+R_{3}(\Psi) 3 u_{2}(\Psi)+R_{4}(\Psi)\right) d \Psi .
$$

We obtain the desired result from the last expression, by substituting the values of $u_{2}(\Psi)$ and $u_{3}(\Psi)$ and integrating, as follows:

$$
\begin{aligned}
u_{4}(\theta)= & \int_{0}^{\theta}\left[R_{2}(\Psi)\left(2 \widetilde{R}_{3}(\Psi)+3\left(\widetilde{R}_{2}(\Psi)\right)^{2}\right)+R_{3}(\Psi) 3 \widetilde{R}_{2}(\Psi)+R_{4}(\Psi)\right] d \Psi \\
= & \left(\widetilde{R}_{2}(\theta)\right)^{3}+2 \int_{0}^{\theta}\left[\widetilde{R}_{3}(\Psi) R_{2}(\Psi)+\widetilde{R}_{2}(\Psi) R_{3}(\Psi)\right] d \Psi \\
& +\int_{0}^{\theta} \widetilde{R}_{2}(\Psi) R_{3}(\Psi) d \Psi+\widetilde{R}_{4}(\theta) \\
= & \left(\widetilde{R}_{2}\right)^{3}+2 \widetilde{R}_{2} \widetilde{R}_{3}+\widetilde{R_{2} R_{3}}+\widetilde{R}_{4} .
\end{aligned}
$$


COROllary 2. The first Lyapunov quantities of (1) are

$$
\begin{aligned}
& V_{3}=\widetilde{R}_{3}(2 \pi), \\
& V_{5}=\left(R_{3}\left(\widetilde{\widetilde{R}_{2}}\right)^{2}+2 \widetilde{R_{4} \widetilde{R}_{2}}+\widetilde{R}_{5}\right)(2 \pi),
\end{aligned}
$$

where the functions $R_{i}(\theta)$ are defined by

$$
\begin{aligned}
R_{2}= & \operatorname{Re} S_{2}, \\
R_{3}= & \operatorname{Re} S_{3}-\operatorname{Re} S_{2} \operatorname{Im} S_{2}, \\
R_{4}= & \operatorname{Re} S_{4}-\operatorname{Re} S_{3} \operatorname{Im} S_{2}+\operatorname{Re} S_{2}\left(\operatorname{Im} S_{2}\right)^{2}-\operatorname{Re} S_{2} \operatorname{Im} S_{3}, \\
R_{5}= & \operatorname{Re} S_{5}-\operatorname{Re} S_{4} \operatorname{Im} S_{2}-\operatorname{Re} S_{2} \operatorname{Im} S_{4}+2 \operatorname{Re} S_{2} \operatorname{Im} S_{2} \operatorname{Im} S_{3} \\
& -\operatorname{Re} S_{3} \operatorname{Im} S_{3}+\operatorname{Re} S_{3}\left(\operatorname{Im} S_{2}\right)^{2}-\operatorname{Re} S_{2}\left(\operatorname{Im} S_{2}\right)^{3},
\end{aligned}
$$

and $S_{k}(\theta)=e^{-i \theta} F_{k}\left(e^{i \theta}, e^{-i \theta}\right)$.

Proof. From the fact that $u_{2}(2 \pi)=0$ and using Proposition 1, we have $\widetilde{R}_{2}(2 \pi)=0$. Hence, the result on $V_{3}$ follows by using Proposition 1 . Assuming that $V_{3}=0$, we get $u_{4}(2 \pi)=0$ and from Proposition 1, again, we get the desired result on $V_{5}$. On the other hand, the expression of $R_{k}$ when $k=2,3,4$ and 5 follows directly from (3). As an example we prove the expression for $R_{4}$. From (3) we have

$$
\begin{aligned}
R_{4} & =\operatorname{Re} S_{4}-\sum_{j=1}^{2} R_{4-j}(\theta) \operatorname{Im} S_{j+1}(\theta) \\
& =\operatorname{Re} S_{4}-\left(\operatorname{Re} S_{3}-\operatorname{Re} S_{2} \operatorname{Im} S_{2}\right) \operatorname{Im} S_{2}-\operatorname{Re} S_{2} \operatorname{Im} S_{3},
\end{aligned}
$$

which gives the expected value of $R_{4}$.

We now recall the following formulas that will be frequently used in the sequel:

$$
\begin{aligned}
& 2 \operatorname{Re} \alpha \operatorname{Re} \beta=\operatorname{Re}[\alpha \beta+\bar{\alpha} \beta], \\
& 2 \operatorname{Im} \alpha \operatorname{Im} \beta=\operatorname{Re}[-\alpha \beta+\bar{\alpha} \beta], \\
& 2 \operatorname{Re} \alpha \operatorname{Im} \beta=\operatorname{Im}[\alpha \beta+\bar{\alpha} \beta], \quad \alpha, \beta \in \mathbb{C} .
\end{aligned}
$$

3. Proof of Theorem A. Firstly we will express the Lyapunov quantities of (1) in terms of the trigonometric polynomials $S_{k}$.

Proposition 3. The first two Lyapunov quantities of system (1) are

$$
\begin{aligned}
V_{3} & =\operatorname{Re} \int_{0}^{2 \pi} S_{3}(\Psi) d \Psi-\frac{1}{2} \operatorname{Im} \int_{0}^{2 \pi} S_{2}^{2}(\Psi) d \Psi, \\
V_{5} & =\operatorname{Re} \int_{0}^{2 \pi} S_{5}(\Psi) d \Psi
\end{aligned}
$$




$$
\begin{aligned}
& -\operatorname{Im} \int_{0}^{2 \pi}\left[T_{2}(\Psi)\left(S_{4}(\Psi)+\bar{S}_{4}(\Psi)\right)+S_{2}(\Psi) S_{4}(\Psi)+\frac{1}{2} S_{3}^{2}(\Psi)\right] d \Psi \\
& +\frac{1}{4} \operatorname{Re} \int_{0}^{2 \pi} S_{3}(\Psi)\left[\left(S_{2}(\Psi)+\bar{S}_{2}(\Psi)\right)^{2}-\left(T_{2}(\Psi)-\bar{T}_{2}(\Psi)+2 S_{2}(\Psi)\right)^{2}\right] d \Psi \\
& +\frac{1}{8} \operatorname{Im} \int_{0}^{2 \pi} S_{2}^{2}(\Psi)\left[T_{2}(\Psi)-\bar{T}_{2}(\Psi)+S_{2}(\Psi)-\bar{S}_{2}(\Psi)\right]^{2} d \Psi
\end{aligned}
$$

where $S_{k}(\psi)=e^{-i \psi} F_{k}\left(e^{i \psi}, e^{-i \psi}\right)$ and $T_{2}(\Psi)=-i\left[\widetilde{S}_{2}(\Psi)-\frac{1}{2 \pi} \int_{0}^{2 \pi} \widetilde{S}_{2}(\theta) d \theta\right]$

Proof. By using Corollary 2 and formulas (5) we get the expression for $V_{3}$.

To obtain $V_{5}$ we recall that by Corollary 2 ,

$$
V_{5}=\left(R_{3}\left(\widetilde{R}_{2}\right)^{2}+2 R_{4} \widetilde{R}_{2}+R_{5}\right) \sim(2 \pi)
$$

In order to simplify the calculations of $V_{5}$ we define, for any real number $v$,

$$
V_{5}(v)=\left(R_{3}\left(\widetilde{R}_{2}+v\right)^{2}+2 R_{4}\left(\widetilde{R}_{2}+v\right)+R_{5}\right)^{\sim}(2 \pi) .
$$

By using the fact that $V_{3}=0\left(\widetilde{R}_{3}(2 \pi)=0\right)$ and also that $u_{4}(2 \pi)=0$ $\left(\left(\widetilde{R}_{4}+\widetilde{R}_{2} R_{3}\right)(2 \pi)=0\right)$, it turns out that $V_{5}(v) \equiv V_{5}$. Therefore we can choose any $v$ for computing $V_{5}$. We choose it such that

$$
\widetilde{R}_{2}+v=\operatorname{Re}\left(\widetilde{S}_{2}+v\right)=\operatorname{Re}\left(i T_{2}\right)=-\operatorname{Im}\left(T_{2}\right) .
$$

Hence

$$
V_{5}=\int_{0}^{2 \pi}\left(R_{3}(\theta)\left(\operatorname{Im}\left(T_{2}(\theta)\right)\right)^{2}-2 R_{4}(\theta) \operatorname{Im}\left(T_{2}(\theta)\right)+R_{5}(\theta)\right) d \theta
$$

To get a more suitable expression for the integrated function we again use Corollary 2, obtaining

$$
\begin{aligned}
\left(\operatorname{Re} S_{3}\right. & \left.-\operatorname{Re} S_{2} \operatorname{Im} S_{2}\right)\left(\operatorname{Im} T_{2}\right)^{2} \\
& -2\left(\operatorname{Re} S_{4}-\operatorname{Re} S_{3} \operatorname{Im} S_{2}+\operatorname{Re} S_{2}\left(\operatorname{Im} S_{2}\right)^{2}-\operatorname{Re} S_{2} \operatorname{Im} S_{3}\right) \operatorname{Im} T_{2} \\
& +\operatorname{Re} S_{5}-\operatorname{Re} S_{4} \operatorname{Im} S_{2}-\operatorname{Re} S_{2} \operatorname{Im} S_{4}+2 \operatorname{Re} S_{2} \operatorname{Im} S_{2} \operatorname{Im} S_{3} \\
& -\operatorname{Re} S_{3} \operatorname{Im} S_{3}+\operatorname{Re} S_{3}\left(\operatorname{Im} S_{2}\right)^{2}-\operatorname{Re} S_{2}\left(\operatorname{Im} S_{2}\right)^{3} .
\end{aligned}
$$

Collecting terms taking into account the number of factors they have, we get

$$
\begin{aligned}
\operatorname{Re} S_{5} & -2 \operatorname{Re} S_{4} \operatorname{Im} T_{2}-\operatorname{Re} S_{4} \operatorname{Im} S_{2}-\operatorname{Re} S_{2} \operatorname{Im} S_{4}-\operatorname{Re} S_{3} \operatorname{Im} S_{3} \\
& +\operatorname{Re} S_{3}\left(\operatorname{Im} T_{2}\right)^{2}+2 \operatorname{Re} S_{3} \operatorname{Im} S_{2} \operatorname{Im} T_{2}+2 \operatorname{Re} S_{2} \operatorname{Im} S_{3} \operatorname{Im} T_{2} \\
& +2 \operatorname{Re} S_{2} \operatorname{Im} S_{2} \operatorname{Im} S_{3}+\operatorname{Re} S_{3}\left(\operatorname{Im} S_{2}\right)^{2} \\
& -\operatorname{Re} S_{2} \operatorname{Im} S_{2}\left(\operatorname{Im} T_{2}\right)^{2}-2 \operatorname{Re} S_{2}\left(\operatorname{Im} S_{2}\right)^{2} \operatorname{Im} T_{2}-\operatorname{Re} S_{2}\left(\operatorname{Im} S_{2}\right)^{3} .
\end{aligned}
$$


Afterwards we will apply iteratively the formulas (5) to arrive at the final expression of $V_{5}$.

Firstly we consider the terms with one, two and three factors. The unique term with exactly one factor is $\operatorname{Re} S_{5}$, and its integral appears in the expression of $V_{5}$. With exactly two factors we have

$$
-2 \operatorname{Re} S_{4} \operatorname{Im} T_{2}-\operatorname{Re} S_{4} \operatorname{Im} S_{2}-\operatorname{Re} S_{2} \operatorname{Im} S_{4}-\operatorname{Re} S_{3} \operatorname{Im} S_{3} .
$$

The use of formulas (5) gives

$$
-\operatorname{Im}\left[T_{2}\left(S_{4}+\bar{S}_{4}\right)+S_{2} S_{4}+\frac{1}{2} S_{3}^{2}\right],
$$

which is the result that appears in the expression of $V_{5}$.

We have the following terms with exactly three factors:

$$
\begin{aligned}
\operatorname{Re} S_{3}\left(\operatorname{Im} T_{2}\right)^{2}+2 \operatorname{Re} S_{3} \operatorname{Im} S_{2} & \operatorname{Im} T_{2}+2 \operatorname{Re} S_{2} \operatorname{Im} S_{3} \operatorname{Im} T_{2} \\
& +2 \operatorname{Re} S_{2} \operatorname{Im} S_{2} \operatorname{Im} S_{3}+\operatorname{Re} S_{3}\left(\operatorname{Im} S_{2}\right)^{2} .
\end{aligned}
$$

Transforming this expression term after term by applying formulas (5), we have

$$
\begin{aligned}
& \operatorname{Re} S_{3}\left(\operatorname{Im} T_{2}\right)^{2}=-\frac{1}{4} \operatorname{Re}\left(S_{3}\left(T_{2}-\bar{T}_{2}\right)^{2}\right), \\
& 2 \operatorname{Re} S_{3} \operatorname{Im} S_{2} \operatorname{Im} T_{2}+2 \operatorname{Re} S_{2} \operatorname{Im} S_{3} \operatorname{Im} T_{2}=-\operatorname{Re}\left(S_{2} S_{3}\left(T_{2}-\bar{T}_{2}\right)\right), \\
& 2 \operatorname{Re} S_{2} \operatorname{Im} S_{2} \operatorname{Im} S_{3}+\operatorname{Re} S_{3}\left(\operatorname{Im} S_{2}\right)^{2}=\frac{1}{4} \operatorname{Re}\left(S_{3}\left[\left(\bar{S}_{2}+S_{2}\right)^{2}-4 S_{2}^{2}\right]\right) .
\end{aligned}
$$

Integrating the sum of the last three expressions we obtain the corresponding term that appears in $V_{5}$.

The computations involving the terms with four factors are tedious but straightforward and we omit them.

As a consequence of the previous proposition we can prove our main result.

Proof of Theorem A. If we express $S_{2}(\theta), S_{3}(\theta), S_{4}(\theta), S_{5}(\theta)$ and $T_{2}(\theta)$ in terms of the coefficients of the differential equation we get

$$
\begin{aligned}
& S_{2}(\theta)=A e^{i \theta}+B e^{-i \theta}+C e^{-3 i \theta}, \\
& S_{3}(\theta)=D e^{2 i \theta}+E+F e^{-2 i \theta}+G e^{-4 i \theta}, \\
& S_{4}(\theta)=H e^{3 i \theta}+I e^{i \theta}+J e^{-i \theta}+K e^{-3 i \theta}+L e^{-5 i \theta}, \\
& S_{5}(\theta)=M e^{4 i \theta}+N e^{2 i \theta}+O+P e^{-2 i \theta}+Q e^{-4 i \theta}+R e^{-6 i \theta}, \\
& T_{2}(\theta)=-A e^{i \theta}+B e^{-i \theta}+\frac{C}{3} e^{-3 i \theta} .
\end{aligned}
$$

To compute $V_{3}$, from Proposition 3 , we need to calculate 


$$
\begin{aligned}
& \operatorname{Re} \int_{0}^{2 \pi}\left(D e^{2 i \theta}+E+F e^{-2 i \theta}+G e^{-4 i \theta}\right) d \theta \\
& \quad-\frac{1}{2} \operatorname{Im} \int_{0}^{2 \pi}\left(A^{2} e^{2 i \theta}+2 A B+\left(B^{2}+2 A C\right) e^{-2 i \theta}+2 B C e^{-4 i \theta}+C^{2} e^{-6 i \theta}\right) d \theta .
\end{aligned}
$$

Hence, it suffices to obtain the terms with no exponential factors. This is because the other terms have $2 \pi$-periodic primitives and consequently, when we integrate between 0 and $2 \pi$, they vanish. Therefore, we have $V_{3}$.

To obtain $V_{5}$, first we obtain the trigonometric polynomial expressions of the integrands in $V_{5}$ of Proposition 3 , and then we utilize the argument used in the calculus of $V_{3}$. That is, we are only interested in the terms of the resulting trigonometric polynomials without exponential factors. This argument allows computing $V_{5}$ by hand. Anyway, observe that by changing $e^{i \theta}$ and $e^{-i \theta}$ to $x$ and $1 / x$ respectively, the problem is reduced to the study of a product of polynomials in $x$ and $1 / x$, which is done extremely fast by computer. In any case, we get the following expression for $V_{5}$ :

$$
\begin{aligned}
V_{5}= & 2 \pi\left[\operatorname{Re}(O)-\operatorname{Im}\left(\frac{1}{2} E^{2}+D F-A \bar{I}+2 B I+B \bar{J}+\frac{4}{3} C H+\frac{1}{3} C \bar{K}\right)\right. \\
& +\frac{1}{4} \operatorname{Re}\left(\frac{32}{9} C \bar{C} E+\frac{8}{3} A C \bar{F}+4 A \bar{B} F+4 B \bar{C} F\right. \\
& -8 B^{2} D-\frac{8}{3} A C D-4 A \bar{B} \bar{D}+\frac{20}{3} B \bar{C} \bar{D} \\
& \left.+\frac{8}{3} A \bar{C} G+\frac{4}{3} B C \bar{G}+4 E \bar{A} \bar{B}+8 B \bar{B} E-4 A B E\right) \\
& +\frac{1}{8} \operatorname{Im}\left(8 A \bar{B}^{2} C+4 A^{2} B^{2}-\frac{16}{3} A^{2} \bar{B} C\right. \\
& \left.\left.+\frac{16}{3} \bar{B}^{3} C-16 A B^{2} \bar{B}-\frac{160}{9} A B C \bar{C}\right)\right]
\end{aligned}
$$

but this expression can be reduced by using the fact that $V_{3}=0$. We note that this fact has already been partially used.

To simplify the expression for $V_{5}$ we proceed as follows. Take the terms

$$
\begin{aligned}
2 \pi\left\{-\operatorname{Im}\left(\frac{1}{2} E^{2}\right)+\frac{1}{4} \operatorname{Re}\left(\frac{32}{9} C \bar{C} E\right.\right. & +4 E \bar{A} \bar{B}+8 B \bar{B} E-4 A B E) \\
& \left.+\frac{1}{8} \operatorname{Im}\left(-16 A B^{2} \bar{B}-\frac{160}{9} A B C \bar{C}\right)\right\} .
\end{aligned}
$$

Using (5) and the fact that $\operatorname{Re}(E)=\operatorname{Im}(A B)$ (i.e. that $V_{3}=0$ ), we have

$$
\begin{gathered}
\frac{1}{4} \operatorname{Re}(8 B \bar{B} E)+\frac{1}{8} \operatorname{Im}\left(-16 A B^{2} \bar{B}\right)=0, \\
2 \pi\left\{\frac{1}{4} \operatorname{Re}\left(\frac{32}{9} C \bar{C} E\right)-\frac{1}{8} \operatorname{Im}\left(\frac{160}{9} A B C \bar{C}\right)\right\}=\frac{-8}{3} \pi C \bar{C} \operatorname{Re}(E),
\end{gathered}
$$

and

$$
2 \pi\left\{-\operatorname{Im}\left(\frac{1}{2} E^{2}\right)+\frac{1}{4} \operatorname{Re}(4 E \bar{A} \bar{B}-4 A B E)\right\}=\pi \operatorname{Im}\left(E^{2}\right) .
$$

Hence, (7) is equal to

$$
2 \pi\left\{\frac{1}{2} \operatorname{Im}\left(E^{2}\right)-\frac{4}{3} \operatorname{Re}(C \bar{C} E)\right\} .
$$

Therefore, by substituting this last expression in (6) we get the final formula for $V_{5}$. 


\section{References}

[AL] M. A. M. Alwash and N. G. Lloyd, Non-autonomous equations related to polynomial two-dimensional systems, Proc. Roy. Soc. Edinburgh Sect. A 105 (1987), 129-152.

[ALGM] A. A. Andronov, E. A. Leontovich, I. I. Gordon and A. G. Maier, Theory of Bifurcations of Dynamic Systems on a Plane, Wiley, New York, 1967.

[CGMM] A. Cima, A. Gasull, V. Mañosa and F. Mañosas, Algebraic properties of the Lyapunov and Period constants, Rocky Mountain J. Math., to appear.

[FLLL] W. W. Farr, C. Li, I. S. Labouriau and W. F. Langford, Degenerate Hopf bifurcation formulas and Hilbert's 16th problem, SIAM J. Math. Anal. 20 (1989), 13-29.

[G] E. Gamero, Computacion simbólica y bifurcaciones de sistemas dinámicos, Ph.D. thesis, Universidad de Sevilla, 1990.

[GW] F. Göbber and K.-D. Willamowski, Ljapunov approach to multiple Hopf bifurcation, J. Math. Anal. Appl. 71 (1979), 333-350.

[GR] I. S. Gradshteyn and I. M. Ryzhik, Tables of Integrals, Series and Products, Academic Press, New York, 1980.

[HW] B. Hassard and Y. H. Wan, Bifurcation formulae derived from center manifold theory, J. Math. Anal. Appl. 63 (1978), 297-312.

Departament de Matemàtiques, Edifici Cc

Universitat Autònoma de Barcelona

08193 Bellaterra, Barcelona, Spain

E-mail: gasull@mat.uab.es

Received on 24.5.1995;

revised version on 15.7 .1996 